\title{
Determination of the Viscoelastic Shear Modulus Using Forced Torsional Vibrations
}

\author{
Edward B. Magrab \\ National Bureau of Standards, Gaithersburg, MD 20899
}

Accepted: December 12, 1983

\begin{abstract}
A forced torsional vibration system has been developed to measure the shear storage and loss moduli on right circular cylindrical specimens whose diameter can vary from 2 to $9 \mathrm{~cm}$ and whose length can vary from 2 to $15 \mathrm{~cm}$. The method and apparatus are usable over a frequency range of 80 to $550 \mathrm{~Hz}$ and a temperature range of $-20^{\circ} \mathrm{C}$ to $80^{\circ} \mathrm{C}$
\end{abstract}

Key words: shear modulus; torsion; vibrations; viscoelastic.

\section{Introduction}

Many methods exist for the experimental determination of the viscoelastic properties of materials. Some of these methods have been summarized $[1,2]^{1}$ and those that have been used more recently appear in a collection of abstracts [3]. The method described herein, which uses forced torsional vibrations, is an updated version of previous works [4,5]. It was selected because it most easily met the requirements placed on the geometry and dimensions of the sample, a circular cylinder whose diameter ranged from 2 to $9 \mathrm{~cm}$ and whose length ranged to $15 \mathrm{cnl}$. This wide range of sizes is a consequence of the desire to use the same sample that was previously subjected to a different kind of material properties' test over a different (higher) frequency range. The method and apparatus described subsequently is usable over a frequency range from 80 to $550 \mathrm{~Hz}$ and a temperature range from $-20{ }^{\circ} \mathrm{C}$ to $80^{\circ} \mathrm{C}$.

About the Author, Paper: Edward B. Magrab is a mechanical engineer in the NBS Automated Production Technology Division. The work on which he reports was sponsored by the U.S. Naval Ship Research and Development Center.

'Figures in brackets indicate literature references at the end of this papcr.

\section{Theory}

Consider the forced harmonic torsional vibrations of a right circular cylinder having a frequency-dependent, complex viscoelastic shear modulus $G^{*}(f)=$ $G^{\prime}(f)+j G^{\prime \prime}(f)$, where $G^{\prime}(f)$ is the shear storagc modulus, $G^{\prime \prime}(f)$ the loss modulus, and $f$ the frequency. If a mass with mass moment of inertia $J$ and a torsional spring of constant $k$ are attached to one end of the cylinder as shown in figure 1, and the other end of the cylinder is subjected to a harmonically varying torque, then the expression for the angular acceleration respouse ratio of the top plane of the cylinder to the bottom plane is given by $[4,5]$

$$
A_{0}=\frac{(\mathrm{Acc})_{\mathrm{TOP}}}{(\mathrm{Acc})_{\mathrm{BOT}}}=\frac{r_{\mathrm{t}}}{r_{\mathrm{b}}}\left[C_{1} \Omega^{*} \sin \left(\Omega^{*}\right)+\cos \left(\Omega^{*}\right)\right]^{-1}
$$

where

$$
\begin{gathered}
C_{1}=\frac{2 J\left[\left(f_{\mathrm{II}} / f\right)^{2}-1\right]}{\pi \rho h\left(b^{4}-a^{4}\right)} \\
\Omega^{*}=x-j y \\
f_{\mathrm{m}}=\frac{1}{2 \pi} \vee \frac{k}{J}
\end{gathered}
$$




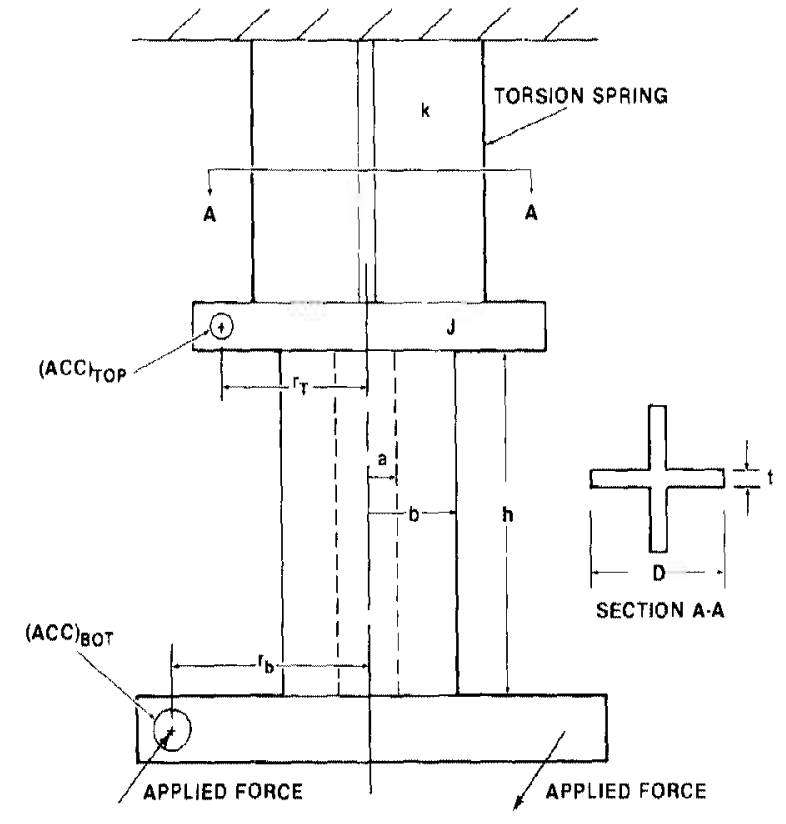

Figure 1-Geometric description of torsion specimen and attached spring and mass.

$$
\begin{gathered}
x=2 \pi \cos (\theta / 2) \frac{\vee f^{2} h^{2}}{p} \\
y=2 \pi \sin (\theta / 2) \sqrt{ } \frac{\rho f^{2} h^{2}}{p} \\
p=\left[G^{\prime 2}+G^{\prime \prime 2}\right]^{1 / 2} \\
\theta=\tan ^{-1}\left(G^{\prime \prime} / G^{\prime}\right)
\end{gathered}
$$

and $\rho$ is the density of the cylinder, $h$ its length, $b$ its outer radius, $a$ its inner radius, and $G^{\prime \prime} / G^{\prime}$ the loss tangent. The quantity $f_{\mathrm{m}}$ is the natural frequency of the attached spring-mass system with the cylindrical specimen removed. The quantity $r_{t}$ is the distance from the center of the axis of the cylinder to the center of the top accelerometer, and $r_{b}$ is the distance from the axis to the point of application of the applied force, which coincides with the location of the bottom accelerometer.

Substituting eq (3) into eq (1) yields

$$
A_{0}=R \mathrm{e}^{j \phi}
$$

where

$$
\begin{gathered}
R=\frac{r_{\mathrm{t}}}{r_{\mathrm{b}}}\left(A^{2}+B^{2}\right)^{1 / 2} \\
\phi=\tan ^{-1}(B / A)
\end{gathered}
$$

and

$A=\left[C_{1} x \sin (x)+\cos (x)\right] \cosh (y)-C_{1} y \cos (x) \sinh (y)$

$B=\left[C_{1} x \cos (x)-\sin (x)\right] \sinh (y)+C_{1} y \sin (x) \cosh (y)$

Thus, if $R$ and $\phi$ are the measured amplitude ratio and phase angle, respectively, and all the physical and geometric parameters of the specimen are determined by other means, then $G^{\prime}$ and $G^{\prime \prime}$ can be found using eqs (6) and (7). However, because of the complexity of these equations, $G^{\prime}$ and $G^{\prime \prime}$ cannot be solved for explicity. The numerical procedure used to obtain these quantities is described in Appendix I.

As the excitation frequency approaches zero and $f \ll f_{\mathrm{m}}$ eq (1) becomes

$$
A_{0}=\left(\frac{r_{\mathrm{t}}}{r_{\mathrm{b}}}\right)\left[1+\frac{C_{0}}{G}\right]^{-1}
$$

where

$$
C_{0}=\left(\frac{8 \pi h}{b^{4}-a^{4}}\right) J f_{m}^{2}
$$

and $G$ is a nominal value for $p$. The range of values for $C_{0}$, in $\mathrm{N} / \mathrm{m}^{2}$, for the experimental setup is approximately $2.5 \times 10^{5} \leqslant C_{0} \leqslant 2 \times 10^{8}$. Thus for materials with a shear modulus of $3 \times 10^{7} \mathrm{~N} / \mathrm{m}^{2}, A_{0}$ will vary from a value slightly less than $r_{b} / r_{b}$ to a value of approximately $\left(r_{t} / r_{b}\right) / 8$

\section{Design Considerations}

\section{General Requirements}

The general requirements are that 1.) the specimens can range in size to approximately $15 \mathrm{~cm}$ in length and $9 \mathrm{~cm}$ in diameter; 2.) the shear moduli can be as low as $3 \times 10^{6} \mathrm{~N} / \mathrm{m}^{2}$; and 3.) the method provides the shear modulus over a broad frequency range from 80 to 550 $\mathrm{Hz}$ and temperature range from $-20^{\circ}$ to $80^{\circ} \mathrm{C}$.

\section{Temperature Considerations}

The temperature requirements place a limit on the overall size of the test fixture, for it has to fit into a temperature chamber of reasonable size. It also has to weigh a modest amount so that one could place it into a chamber without the chamber requiring additional 
structural support. Lastly, in order to operate the vibration cxciters over the temperature range, the exciters have to be air cooled/heated with room temperature air $\left(20^{\circ} \mathrm{C}\right)$ so that the springs that sustain the exciter's moving element retain their desired properties.

The temperature of the specimen is determined from the placement of a thermocouple on the specimen's surface and is read by a digital output device with an accuracy of $\pm 1{ }^{\circ} \mathrm{C}$.

\section{Fixture}

According to the theory used to determine the shear modulus from the experimentally determined data, the specimen should be subjected to torsional motion only, with no bending of the specimen. In addition, the fixture itself must be free from structural resonances over a broad frequency range and for test samples of varying length and diameter. The design chosen was a combination of certain features of previous works $[4,5]$ and is shown in figure 2.

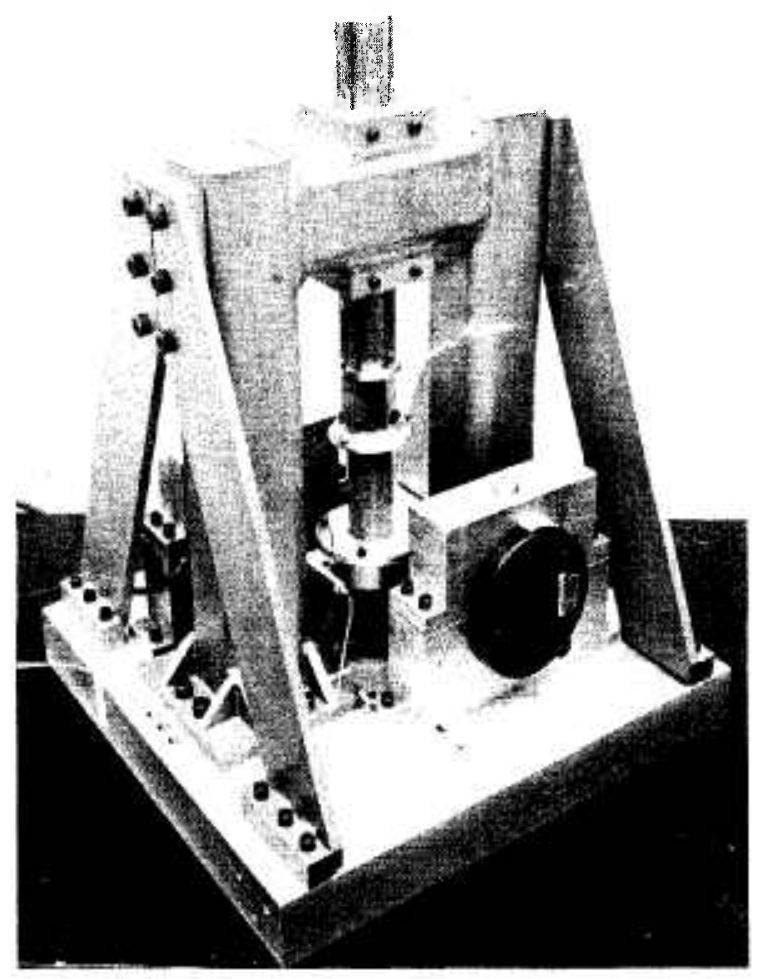

Figure 2-Forced torsional vibration apparatus.

To minimize bending the top and bottom torsional springs have an " $x$ " cross-section, which is very stiff in bending compared to its twisting resistance. To show this consider a single force $F$ acting on the spring through a moment arm about the center of the spring of radius $r_{\mathrm{b}}$. The displacement due to bending, $s_{B}$, is

$$
s_{B}=\frac{1}{3} \frac{F L^{3}}{E I}
$$

and that due to rotation

$$
s_{1}=\frac{F r_{b}^{2} L}{K G}
$$

where $G$ and $E$ are the shear and tensile moduli of the spring, respectively, $L$ is its length and $I$ and $K$ are the moment of inertia and the torsion constant of the crosssection, respectively. The accelerations of the spring are proportional to these displacements. Thus a good measure of their relative resistance to this unbalanced torque is their ratio. Hence,

$$
\frac{s_{B}}{s_{T}}=\frac{1}{6(1+v)}\left(\frac{L}{r_{\mathrm{b}}}\right)^{2} \frac{K}{I}
$$

where $v$ is Poisson's ratio. For the " $\mathrm{x}$ " cross-section shown in figure 1,

$$
\begin{aligned}
& K \cong \frac{2}{3} D t^{3} \\
& I \cong \frac{t D^{3}}{12} .
\end{aligned}
$$

Therefore,

$$
\frac{s_{B}}{s_{T}}=\left(\frac{L}{r_{\mathrm{b}}}\right)^{2}\left(\frac{t}{D}\right)^{2}
$$

The bottom spring has the following dimensions: $L=8.26 \mathrm{~cm}, r_{\mathrm{b}}=7.46 \mathrm{~cm}, t=0.32 \mathrm{~cm}$, and $D=10.16 \mathrm{~cm}$. Using these values in eq (11) yields that $s_{B} / s_{T} \cong 0.001$. Thus, the bending displacements can be expected to be on the order of a thousand times less than the desired torsionally induced displacements. In the actual system two dynamic exciters are used, which eliminates most of the unbalanced force. The output force of the shakers, however, is not controlled, and it is assumed that the same input voltage to both exciters yields approximately the same output force. However, because of this great difference in stiffnesses, any small imbalance docs not strongly couple to the system. For the top spring the parameters in eq (10) are: $L \cong 5.08 \mathrm{~cm}, t=0.0794 \mathrm{~cm}$, and $D=6.99 \mathrm{~cm}$. Equation (10) now yields $s_{B} / s_{T} \cong 0.0002$. 
The experimental confirmation of the excitation portion of the fixture's resistance to bending is shown in figure 3. The input signal to the shakers was broadband random noise. A commercial digital frequency analyzer was used to obtain the time-averaged transfer function of the input voltage to the shakers to the voltage from the bottom accelerometer. The result is shown in figure 3. The absence of any resonance peaks until approximately $680 \mathrm{~Hz}$, except the one dircctly related to thc first torsional resonance of spring-mass system, can be seen. This indicates that the useful frequency range of the fixture is as high as approximately $550 \mathrm{~Hz}$. The torsional natural frequency of the bottom spring mass system is of no consequence in the experiment because the magnitude of the input acceleration is kept relatively constant over the entire test frequency range.

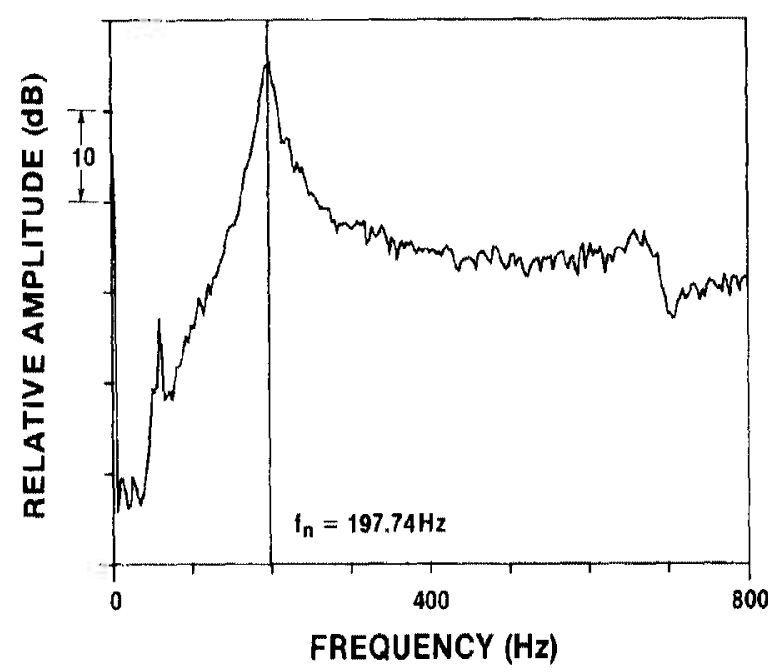

Figure 3-Transfer function of bottom torsional spring and attached mass.

\section{Determination of $J$ and $f_{\mathrm{m}}$}

The top end of the top torsion spring is bolted to a movable solid steel cylinder as shown in figure 2. According to the theory developed previously, this connection must be rigid compared to the torsion spring itself. The ratio of the torsional rigidity of the solid cylinder, $K_{\mathrm{c}}$, to that of the torsion spring, $K$ (given by eq (10)), is

$$
\frac{K_{c}}{K}=\frac{3 \pi}{64}\left(\frac{D}{t}\right)^{3}
$$

Substituting in the appropriate dimensional values for the fixture shown in figure 2 yields $K_{c} / K \cong 10^{5}$. Thus, the assumption of rigidity is a good one.
Another important consideration is the range of values for $J$, the mass moment of inertia of the attached mass. For a given natural frequency of this system the specimen, when connected to $J$, must be able to influence both the value of $f_{\mathrm{m}}$ and, more importantly, its damping. An indication of the magnitude of the parameters that influence these properties can be approximately determined by assuming that the vibrating system consists of the inertia $J$ connccted to two springs; one is the " $x$ " cross-section torsion spring already discussed and the other is the spring formed by the torsional resistance of the specimen, assuming that its internal damping can be ignored in this portion of the analysis. The natural frequency of this new system, $f_{\mathrm{m}}^{\prime}$, is then given by

$$
f_{\mathrm{m}}^{\prime}=f_{\mathrm{m}}\left[1+C_{2}\right]^{1 / 2}
$$

where $f_{\mathrm{m}}$ is given by eq (3) and

$$
C_{2}=\frac{\pi}{2}\left(\begin{array}{l}
G \\
h
\end{array}\right) \frac{\left(b^{4}-a^{4}\right)}{\left(2 \pi f_{m}\right)^{2}}\left(\begin{array}{l}
1 \\
J
\end{array}\right)
$$

It is seen that in order for $C_{2}$ to be greater than one, the combination of $h, f_{\mathrm{m}}$, and $J$ must be chosen carefully for a given material. Unfortunately the class of materials that is to be tested is very weak in torsion; typically these materials have a shear modulus of 4 to $15 \times 10^{6} \mathrm{~N} / \mathrm{m}^{2}$. Consequently the design requires that $J, h$, and $f_{\mathrm{m}}$ be as small as possible. There are, of course, some physical limitations in just how small $J$ can be made. In the apparatus shown in figure $2, J=1.8972 \mathrm{~kg}-\mathrm{cm}^{2}$. The computational proccdure to obtain $J$ is given in Appendix II.

Whereas $J$ was computed, the natural frequency of the attached spring/mass system, $f_{m}$, without the test specimen attached was determined experimentally by using a digital frequency analyzer in its transfer function mode. The excitation was applied with a hammer to one of the "ears" (see figs. 2 and A-2) of the top mass. The noncontacting end of the hammer had an accelcrometer mounted to it. The resulting motion of the spring/mass was recorded by the accelerometer mounted on one of the "ears." Using the zoom capability of the analyzer, $f_{\mathrm{m}}$ was determined to within $\pm 0.25 \mathrm{~Hz}$, which, for natural frequencies $f_{\mathrm{m}}>100 \mathrm{~Hz}$ results in an uncertainty of $0.25 \%$ or less. The results for two torsion springs, one with $t=0.7938 \mathrm{~mm}$ and the other $t=1.588 \mathrm{~mm}$ (see fig. 1) and for two thickness of the acceleromcter mounting disk (see fig. A-2) are summarized in table 1. From the 
Table 1. Values of $f_{\mathrm{m}}$ for four combinations of springs and inertias $(J)$.

\begin{tabular}{lcc}
\hline & \multicolumn{2}{c}{$f_{\mathrm{th}}(\mathrm{Hz})$} \\
\cline { 2 - 3 } $\begin{array}{l}\text { Spring flange } \\
\text { thickness (nm) }\end{array}$ & $\begin{array}{l}J=1.8972 \mathrm{~kg}-\mathrm{cm}^{2} \\
\left(h_{A}=6.35 \mathrm{~mm}\right)\end{array}$ & $\begin{array}{c}J=2.3652 \mathrm{~kg}-\mathrm{cm}^{2} \\
\left(h_{4}=12.75 \mathrm{~mm}\right)\end{array}$ \\
\hline 0.7938 & 100.75 & 89.00 \\
1.588 & 283.00 & 255.00
\end{tabular}

discussion in the preceding paragraph it is seen that for the weaker class of materials the spring/mass combinations resulting in the lower set of $f_{\mathrm{m}}$ should be used.

\section{Specimen Mounting}

In order to niount the specimen concentrically with respect to the top and bottom springs and to climinate any pre-twist of the specimen, the technique employed used two discs, one $12.3 \mathrm{~cm}$ in diameter and attached to the bottom "ears" and the other $6.9 \mathrm{~cm}$ in diameter and attached to the top "ears" (and included in the calculation of J), each having on one of its faces a concentric raised disk $1.5 \mathrm{~mm}$ ligh and $12.7 \mathrm{~mm}$ in diameter. The disks are mounted on their respective bell cranks with the raised disks facing each other. Prior to mounting the cylindrical specimen, the specimen is placed in a lathe to have each of its end planes turned smooth and perpendicular to its axis. In addition, a concentric cylindrical depression is turned on each of the end planes that is 1.59 $\mathrm{mm}$ deep and $12.7 \mathrm{~mm}$ in diameter. Epoxy is applied to each end plane of the cylindrical specimen and the specimen is placed onto the bottom disk. The top spring mass assembly is then lowered onto the top of the specimen and the epoxy is allowed to cure.

\section{Instrumentation}

The computer controlled instrumentation system used to measure the accelerometers' amplitude ratio and phase angle is shown in figure 4 . The electrodynamic vibration exciters are connected in parallel and receive their input voltage from an amplifier with the capacity to provide $15 \mathrm{~A} \mathrm{rms}$ into $1 \mathrm{ohm}$. The input to the amplifier is connected to an oscillator. The exciters themselves have an impedance of approximately 2 ohms and require $5 \mathrm{~A} \mathrm{rms}$ to obtain their rated output force. The oscillator's output voltage amplitude and frequency are under computer control.

The accelerometers have a sensitivity of nominally 10 $\mathrm{mV} / \mathrm{g}$ and have a unity gain preamplifier built into them. The output signals from the accelerometers are then amplified $30 \mathrm{~dB}$ and passed through two $2 \mathrm{~Hz}$ bandwidth tracking filters. The tracking frequency is provided by a second output signal from the oscillator which remains constant at $1 \mathrm{~V}$. This $1 \mathrm{~V}$ signal is amplified to mcct the tracking filter's requirement of $3.5 \mathrm{~V}$. The $30 \mathrm{~dB}$ gain given to each accelerometer signal provides better use of the dynamic range of the tracking filters and uses the digital phasemeter in a voltage range in which its accuracy is better, namely, at levels above $50 \mathrm{mV}$ rms. To maintain control over the voltage levels throughout the electronic measuring system, the output voltage of the oscillator is continuously adjusted so that the output voltage of the bottom accelerometer remains approximately constant at $2.5 \mathrm{mV}$ rms over the entire test frequency range.

The signals from the output of the tracking filters are sent directly to the digital phasemeter and the digital voltmeter, both of which are under computer control. However, the signals to the voltmeter are read sequentially witl the aid of a computer controlled switching setup. The phasemeter has an autocalibration feature which is employed every sixth measurement to ensure that the phase measurements are as precise as possible. The digital voltmeter is used in its autoranging mode, since the output voltage for the upper accelerometer differs widely over the frequency range.

The purpose for the attenuator (labeled ATTN) is discussed in detail subsequently.

To accurately measure the acceleration ratio of the top accelerometer to thc bottom one and the phase angle between them, one must remove from the measured data the influences of any electronics inserted between the electrical signal at the output of the accelerometers and the recording instruments: the digital voltmeter and the digital phasemeter. The ultimate accuracy of the measurement depends, of course, on the accuracy of the voltmeter and the phasemeter. In this experiment the voltmeter has an accuracy of approximately $\pm 0.2 \mathrm{~dB}$ for rms voltage above $100 \mathrm{mV}$ and $\pm 0.3 \mathrm{~dB}$ for voltages less than $100 \mathrm{mV}$ and greater than $100 \mu \mathrm{V}$ over the frequency range 1 to $2000 \mathrm{~Hz}$. The phasemeter has an accuracy of $\pm 0.1^{\circ}$ for rms voltages greater than $50 \mathrm{mV}$ and $\pm 0.2^{\circ}$ for rms voltages as low as $1 \mathrm{mV}$ from $50 \mathrm{~Hz}$ to $50 \mathrm{KHz}$.

The effects of the intervening electronics are removed in the following manner. Consider the simplified equipment diagram given in figure 5 . Let $H,(f)$ be the transfer function of the accelerometer's built-in impedance converters, $H_{0 j}$ the transfer function of the $30 \mathrm{~dB}$ fixed gain amplifiers, and $H_{F j}$ the transfer function of the tracking filter, where $j=1$ refers to the bottom accelerometer channel and $j=2$ to the top channel. The true 
Figure 4-Schematic of complete instrumentation system.

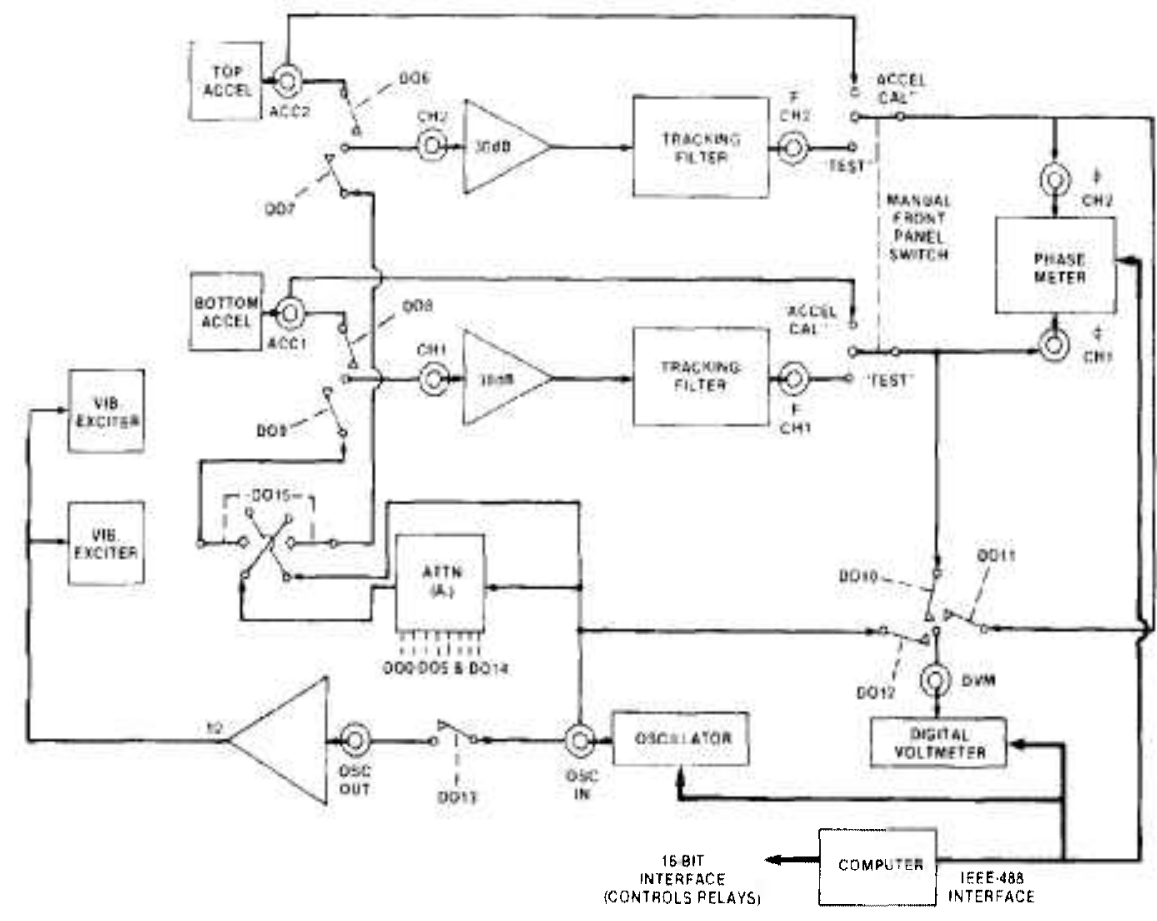

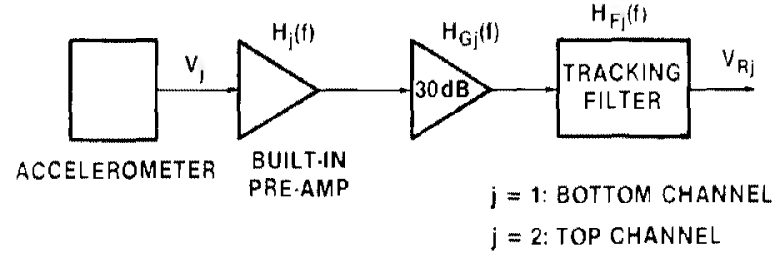

Figure 5-Simplified version of a portion of the instrumentation system.

output signals from the accelerometers, $V_{j}$, are related to the signals appearing at the inputs to the digital voltmeter and phasemeter, $V_{R j}$, as follows:

$$
V_{R j}=V_{j} H_{j} H_{G j} H_{F i}, j=1,2 \text {. }
$$

Solving eq (14) for the acceleration ratio $A_{0}=V_{2} / V_{1}$ yields

$$
\frac{V_{2}}{V_{1}}=\left(\frac{V_{R 2}}{V_{R 1}}\right)\left(\frac{1}{C}\right)
$$

where $C$ is a complex quantity given by

$$
C=\left(\frac{H_{2}}{H_{1}}\right)\left(\frac{H_{G 2} H_{F 2}}{H_{G 1} H_{F 1}}\right)
$$

Thus, the amplitude ratio and phase angle are, respectively,

$$
\begin{gathered}
\left|\frac{V_{2}}{V_{1}}\right|=\left|\frac{V_{R 2}}{V_{R 1}}\right|\left|\frac{1}{C}\right| \\
\phi_{21}=\phi_{R 21}-\phi_{c}
\end{gathered}
$$

where $\left|V_{R 2} / V_{R 1}\right|$ is the ratio of the values read from the digital voltmeter and $\phi_{R 21}$ is the value read from the digital phasemeter. The quantity $\left|C^{-1}\right|$ is the correction that must be isolated and measured in order to remove the influence of the electronic components on the amplitude measurement and $\phi_{c}$ is the quantity that must be isolated and measured to remove the influence on the phase angle measurement.

To remove the effects of $C$ at a given temperature, we proceed by first removing $H_{G i}$ and $H_{E j}$ from the measurement chain and then performing a back-to-back calibration of the two accelerometers. Thus, $V_{2}=V_{1} \mathrm{e}^{j \pi}$ (since one accelerometer is upside down with respect to the other) and

$$
\begin{gathered}
\left|\frac{H_{2}}{H_{1}}\right|=\left|\frac{V_{R 2}}{V_{R 1}}\right|_{B B} \\
\phi_{21}=\phi_{B B}+\pi
\end{gathered}
$$

where $\left|V_{\mathrm{R} 2} / V_{\mathrm{R} 1}\right|$ is determined directly from the reading of the digital voltmeter and $\phi_{B B}$ directly from the 
digital phasemeter. A picture of the back-to-back calibration fixture is shown in figure 6 .

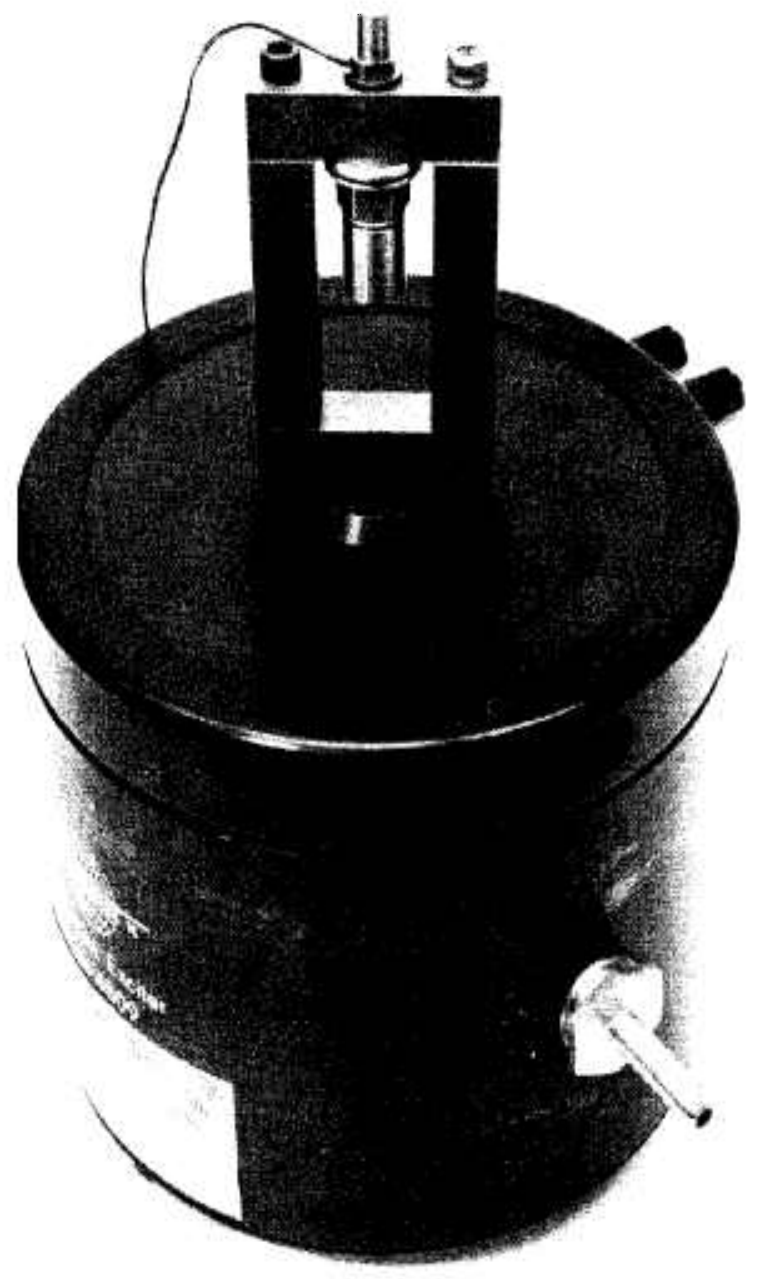

Figure 6-Back-to-back calibration fixture

We now disconnect the accelerometers from the measurement chain and replace them by two signals at the same frequency that differ only in amplitude and not phase. Thus $V_{1} H_{1}=V_{1}$ and $V_{2} H_{2}=A_{1} V_{1}$ where $V_{1}$ is the amplitude of the input voltage to both channels and $0<A_{t}<1$. The attenuation $A_{l}$ is introduced because of the high degree of amplitude and phase nonlinearity of the tracking filters. The introduction of the attenuator allows the input signals to each channel to be approximately equal (to within $\pm 2.5 \mathrm{~dB}$ ) to each accelerometer's output signal. In the actual test procedure the attenuation $A_{t}$ can be introduced to either channel depending on whether or not the accelerometer's output signal ratio is greater than or less than 1 . If the $A_{t}$ is switched, then $A_{t}$ is simply replaced by $1 / A_{t}$ in the subsequent results. Equation (17) then yields

$$
\begin{gathered}
\left|\frac{H_{G 2} H_{F-2}}{H_{G 1} H_{F 1}}\right|=A_{t}\left|\frac{V_{R 2}}{V_{R 1}}\right|, \\
\phi_{21}=\phi_{l}
\end{gathered}
$$

where $\left|V_{R_{2}} / V_{R_{1}}\right|_{I}$ is determined directly from the readings of the digital voltmeter and $\phi$, directly from the digital phasemeter. Using eqs (18) and (19) yields the desired result

$$
\begin{gathered}
\left.\left|\begin{array}{c}
\left|\frac{V_{2}}{V_{1}}\right| \\
\mid
\end{array}=\right| \frac{V_{R 2}}{V_{R 1}} \mid\left[A_{t}\left|\frac{V_{R 2}}{V_{R 1}}\right|_{B B} \mid \begin{array}{l}
V_{R 2} \\
V_{R 1}
\end{array}\right]_{I}\right]^{-1} \\
\phi_{21}=\phi_{R 21}-\phi_{R B}-\pi-\phi_{I}
\end{gathered}
$$

The test procedures used to determine the quantities in eq (20) are done under computer control using a set of 16 rclays.

In the actual testing of the accelerometer's back-toback response it was found that at a given temperature it could be assumed that the accelerometers' relative amplitudes are constant over the frequency range of interest and that the phase difference was essentially zero. In other words $\left|V_{R 2} / V_{R 1}\right|_{B B}=C_{3}$, a constant and $\phi_{n B}=0 \mathrm{in} \mathrm{eq}$ (18). The constant $C_{3}$ is determined at each temperature at a nominal frequency of $200 \mathrm{~Hz}$.

The errors assoctated with the measurement of the amplitude ratio and phase angle cannot be easily related to the resulting errors in the determination of the shear modulus because of the highly nonlinear nature of eqs (5) to (7). Consequently, to get an estimate of the accuracy of the numerically evaluated $G^{\prime}$ and $G^{\prime \prime}$, the following procedure is used. Equation (1) is evaluated over a frequency range for an assumed value of $G^{\prime}$ and $G^{\prime \prime}$. This results in a set of amplitude ratios $\left(A_{0}\right)$ and phase angles $(\phi)$ as a function of frequency. The set of $A_{0}$ and $\phi$ is then altered by the accuracy of the digital voltmeter and phasemeter, and the inverse problem is considered. Thus, at a given frequency we change the anplitude ratios by up to $\pm 0.2 \mathrm{~dB}$ and the phase angle by up to $\pm 0.3^{\circ}$ and solve eq (11) for $G^{\prime}$ and $G^{\prime \prime}$. This procedure was used for $J=1.9 \mathrm{~kg}-\mathrm{cm}^{2}, f_{\mathrm{m}}=100 \mathrm{~Hz}, \rho=1000 \mathrm{~kg} /$ $\mathrm{m}^{3}, G^{\prime}=20 \times 10^{6} \mathrm{~N} / \mathrm{m}^{2}, G^{\prime \prime} / G^{\prime}=0.4, h=10 \mathrm{~cm}, b=5$ $\mathrm{cm}$, and $a=0$. It was found that the percentage errors in $G^{\prime}$ and $G^{\prime \prime} / G^{\prime}$ are small $(<5 \%)$ and asymmetrical with respect to the errors in the amplitude and phase at frequencies away from $f_{\mathrm{m}}$ and large and unequal at frequencies at or near $f_{\mathrm{m}}$, with the largest errors oceurring in the loss tangent ( $\pm 15 \%)$

Another inaccuracy in the determination of the values for $G^{\prime}$ and $G^{\prime \prime}$ are errors due to the calculation of $J$ and the determination of $f_{\mathrm{m}}$ in eq (2). Using a procedure similar to that done previously showed that the effects 
of $J$ and $f_{\mathrm{m}}$ was less than $\pm 3 \%$ except at $f=100 \mathrm{~Hz}$ where it was $\pm 6 \%$. There are no effects of the errors in $J$ and $f_{\mathrm{m}}$ on the ratio $G^{\prime \prime} / G^{\prime}$.

\section{Results}

The apparatus and computer controlled test procedure were used to determine the complex shear modulus of an inhomogeneous polyurethane material with $4 \%$ of its volume containing air bubbles. The sample was $5.08 \mathrm{~cm}$ in diameter and its original height was 10.16 $\mathrm{cm}$. Sevcral different combinations of measurements were made to get an estimate of the variability of the complex shear modulus values as a function of slight changes in specimen temperature, choice of $f_{\mathrm{m}}$, and the height of the specimen. To determine the latter effect the original $10.16-\mathrm{cm}$-high specimen was cut into two pieces, one $4.8 \mathrm{~cm}$ high and the other $4.9 \mathrm{~cm}$ high. The results are tabulated in tables 2 and 3 .

Using both tables and comparing the results of the data in coluinn 1 with those in column 2, those of column 3 with those of 4,5 with 6 , and those of column 7 with those of 8 , it is seen that the repeatability of the data is excellent-typically better than $1 \%$ for both the storage modulus and the loss tangent. Comparison of col- umns 3-6 of both tables indicates that the effect of a choice of $f_{m}$ also yields a repeatibility of better than $1 \%$ for the storage modulus and $8 \%$ for the loss tangent. The large variation in the loss tangent is consistent with the results found from the error analysis described in the previous section

In comparing columns 1 and 2 with columus 3 and 4 and with columns 7 and 8 , it is seen that the change in length of the specimen yields results that are within $4 \%$ of each other. This small deviation is probably due to the unequal distribution of the air bubbles within the material.

Comparing columns 7 and 8 with column 9 shows the change in values of the moduli due to the change in the test site. Although the loss tangent seemed to remain unchanged, the shear storage modulus inexplicably decreased by about $7 \%$.

The apparatus and test method produce data that are self-consistent. In addition, when the results are compared to values obtaincd previously from other tests at much higher frequencies, but on the same sample, these values are what are expected.

The 4.8-cm-high specimen was tested over a range of temperatures from $-10^{\circ} \mathrm{C}$ to $40^{\circ} \mathrm{C}$ with $f_{\mathrm{m}}=100.8 \mathrm{~Hz}$. The results are shown in figurcs 7 and 8 where they

Table 2. Variability of the storage modulus $\left(G^{\prime}\right)$ as a function of several test parameters.

\begin{tabular}{|c|c|c|c|c|c|c|c|c|c|}
\hline & 1 & 2 & 3 & 4 & 5 & 6 & 7 & 8 & 9 \\
\hline Length (cm) & 10.16 & 10.16 & 4.8 & 4.8 & 48 & 4.8 & 4.9 & 4.9 & 4.9 \\
\hline$f_{\mathrm{m}}(\mathrm{Hz})$ & 100.8 & 100.8 & 100.8 & 100.8 & 89.0 & 89.0 & 100.8 & 100.8 & 100.8 \\
\hline Temperature $\left({ }^{\circ} \mathrm{C}\right)$ & 24.0 & 24.2 & 21.2 & 21.9 & 23.2 & 23.2 & 21.1 & 22.3 & 22.6 \\
\hline Date & 1 Sept 82 & 1 Sept 82 & 7 Sept 82 & 7 Sept 82 & 7 Sept 82 & 7 Sept 82 & 3 Sept 82 & 3 Sept 82 & 15 Sept 82 \\
\hline Time & $11: 39$ & $12: 18$ & $9: 36$ & $10: 27$ & $14: 52$ & $15: 49$ & $9: 29$ & 11:09 & $15: 11$ \\
\hline Frequency $(\mathrm{Hz})$ & & & & & $\gamma^{\prime}\left(\mathrm{N} / \mathrm{m}^{2}\right)$ & & & & \\
\hline 100 & & & 14.28 & 13.57 & 15.66 & 15.52 & & & 12.87 \\
\hline 110 & 14.51 & 14.45 & 14.13 & 14.06 & 15.33 & 15.27 & 18.46 & 18.32 & 12.97 \\
\hline 120 & 14.58 & 14.53 & 15.18 & 15.43 & 14.84 & 14.84 & 16.47 & 16.48 & 13.88 \\
\hline 130 & 14.36 & 14.33 & 14.51 & 14.43 & 14.92 & 14.91 & 15.45 & 15.39 & 13.38 \\
\hline 140 & 14.38 & 14.56 & 14.82 & 14.66 & 15.13 & 15.14 & 15.49 & 15.33 & 13.55 \\
\hline 150 & 14.65 & 14.70 & 15.11 & 14.97 & 15.12 & 15.13 & 15.70 & 15.48 & 14.42 \\
\hline 160 & 14.93 & 14.86 & 15.09 & 14.91 & 15.12 & 15.12 & 15.62 & 15.41 & 14.54 \\
\hline 170 & 15.32 & 15.25 & 14.93 & 14.75 & 15.22 & 15.22 & 15.48 & 15.30 & 14.55 \\
\hline 180 & 15.27 & 15.11 & 14.94 & 14.72 & 15.34 & 15.35 & 15.52 & 15.30 & 14.36 \\
\hline 190 & 15.63 & 15.57 & 15.09 & 14.88 & 15.46 & 15.46 & 15.66 & 15.49 & 14.47 \\
\hline 200 & 15.66 & 15.53 & 15.11 & 15.01 & 15.55 & 15.57 & 15.74 & 15.61 & 14.54 \\
\hline 210 & & & 15.30 & 15.20 & 15.82 & 15.82 & 15.92 & 15.84 & 14.64 \\
\hline 220 & & & 15.61 & 15.52 & 15.94 & 15.94 & 16.05 & 15.96 & 14.92 \\
\hline 230 & & & 16.26 & 16.18 & 16.43 & 16.45 & 16.30 & 16.17 & 15.27 \\
\hline 240 & & & 16.54 & 16.47 & 16.72 & 16.70 & 16.43 & 16.31 & 15.18 \\
\hline 250 & & & 16.54 & 16.42 & 16.96 & 16.99 & 16.39 & 16.27 & 15.33 \\
\hline 260 & & & 16.77 & 16.64 & 17.39 & 17.40 & 16.63 & 16.51 & 15.47 \\
\hline l'est Site: & \multicolumn{8}{|c|}{ NBS } & NSRDC \\
\hline
\end{tabular}


Table 3. Variability of the loss tangent $\left(G^{\prime \prime}, G^{\prime}\right)$ as function of several test parameters.

\begin{tabular}{|c|c|c|c|c|c|c|c|c|c|}
\hline & 1 & 2 & 3 & 4 & 5 & 6 & 7 & 8 & 9 \\
\hline $\begin{array}{l}\text { Length }(\mathrm{cm}) \\
f_{\mathrm{m}}(\mathrm{Hz}) \\
\text { Temperature }\left({ }^{\circ} \mathrm{C}\right) \\
\text { Date } \\
\text { Time }\end{array}$ & $\begin{array}{c}10.16 \\
100.8 \\
24.0 \\
1 \text { Sept } 82 \\
11: 39\end{array}$ & $\begin{array}{c}10.16 \\
100.8 \\
24.2 \\
1 \text { Sept } 82 \\
12: 18\end{array}$ & $\begin{array}{c}4.8 \\
100.8 \\
21.2 \\
7 \text { Sept } 82 \\
9.36\end{array}$ & $\begin{array}{c}4.8 \\
100.8 \\
21.9 \\
7 \text { Sept } 82 \\
10: 27\end{array}$ & $\begin{array}{c}4.8 \\
89.0 \\
23.2 \\
7 \text { Sept } 82 \\
14.52\end{array}$ & $\begin{array}{c}4.8 \\
89.0 \\
23.2 \\
7 \text { Sept } 82 \\
15.49\end{array}$ & $\begin{array}{c}4.9 \\
100.8 \\
21.1 \\
3 \text { Sept } 82 \\
9: 29\end{array}$ & $\begin{array}{c}4.9 \\
100.8 \\
22.3 \\
3 \text { Sept } 82 \\
11: 09\end{array}$ & $\begin{array}{c}4.9 \\
100.8 \\
22.6 \\
15 \text { Sept } 82 \\
15: 11\end{array}$ \\
\hline Frequency (fiz) & & & & & $G^{\prime \prime} / G^{\prime}$ & & & & \\
\hline 100 & & & .269 & .173 & .125 & 140 & & & .703 \\
\hline 110 & .224 & .222 & .360 & .310 & .184 & .180 & .238 & .203 & .406 \\
\hline 120 & .229 & .227 & .302 & .249 & .200 & .204 & .275 & .269 & .352 \\
\hline 130 & .230 & .226 & .250 & .249 & .210 & .211 & .231 & .237 & .320 \\
\hline 140 & .260 & .287 & .252 & .250 & .220 & .220 & .240 & .238 & .259 \\
\hline 150 & .255 & .263 & .254 & .252 & .218 & .218 & .247 & .246 & .226 \\
\hline 160 & .255 & .256 & .256 & .253 & .220 & .220 & .254 & .252 & .217 \\
\hline 170 & .241 & .244 & .253 & .252 & .210 & .210 & .246 & .243 & .222 \\
\hline 180 & .253 & .223 & .247 & .249 & .211 & .211 & .235 & .230 & .237 \\
\hline 190 & .224 & .228 & .260 & .256 & .215 & .215 & .237 & .232 & .240 \\
\hline 200 & .245 & .248 & .275 & .271 & .220 & .218 & .243 & .241 & .245 \\
\hline 210 & & & .287 & .287 & .232 & .231 & .241 & .241 & .249 \\
\hline 220 & & & .303 & .302 & .245 & .245 & .243 & .240 & .246 \\
\hline 230 & & & .291 & .287 & .245 & .244 & .242 & .243 & .249 \\
\hline 240 & & & .278 & .275 & .241 & 243 & .247 & .248 & 254 \\
\hline 250 & & & .280 & .281 & .238 & .236 & .251 & .249 & .253 \\
\hline 260 & & & .279 & .278 & .228 & .226 & .250 & .250 & .255 \\
\hline
\end{tabular}

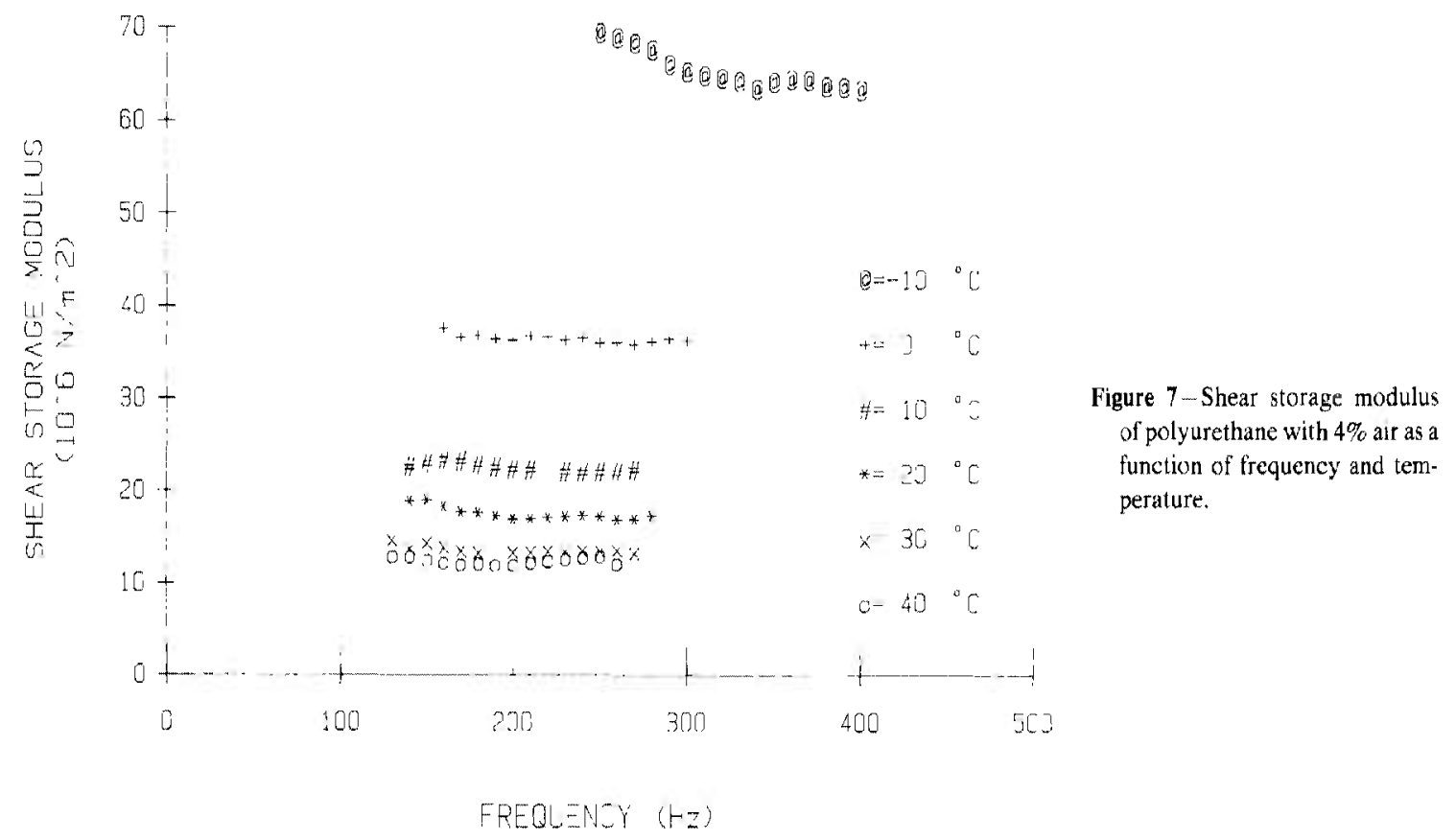




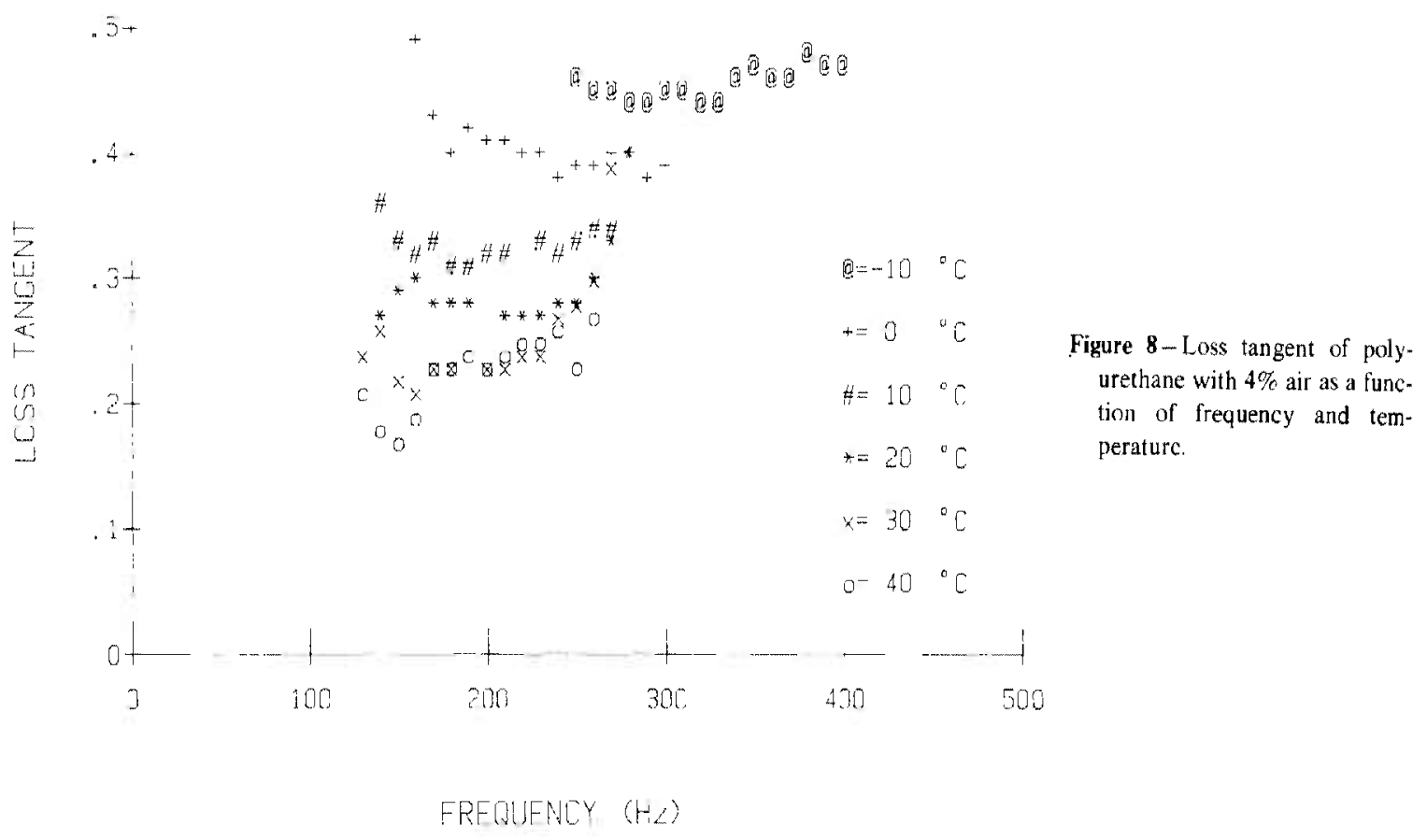

exhibit the desired properties of high loss tangent at decreasing temperatures and of being relatively frequency independent. Unfortunately, from a rheological point of view this material does not exhibit the property of time-temperature equivalence and therefore cannot be reduced to a composite master curve that would effectively produce shear moduli data at a given temperature over a much greater frequency range than shown.

\section{References}

[1] Ward, I. M. Mechanical Properties of Solid Polymers, London: John Wilcy and Sons (1971). Chapter 6.

[2] Cremer, L.; M. Heckl and E. E. Unger, Structure-Borne Sound, Berlin: Springer-Verlag (1973), pp. 189-216.

[3] Measurement of dymamic modul and loss factors of viscoelastic materials, Session J, 99th Meeting: Acoustical Socicty of America, J. Acoust. Soc. of Amer., Suppl. 1, Vol. 67, spring 1980, pp. S23-S25.

[4] Gottenberg, W. J, and R. M. Christensen. An experiment for the determination of the mechanical property in shear for a linear, isotropic viscoelastic solid, Int. J. Engng. Sci., Vol. 2 (1964), pp. 45-57.

[5] Baltrukonis, J. H.; D. S. Blomquist and E. B. Magrab. Measurement of the complex shear modulus of a linearly viscoelastic material. Technical Report \#5, Department of Engineering Mechanics, Catholic University of America, Washington, D.C. (May 1964).

The assistance of William Penzes, for his design and construction of the switching network, $30 \mathrm{~dB}$ amplifiers, and various power supplies and connectors, is greatfully acknowledged.

R. Reitz of the U.S. Naval Research and Development Center obtained the data presented in figures 7 and 8 .

[6] Marquardt, D. W. An algorithm for least squares estimation on nonlinear parameters, J. Soc. Ind. Appl. Math, Vol. II (1963), pp. $431-441$

[7] Nash, J. C. Compact Numerical Methods for Computers: Linear Algebra and Functiun Minimization, New York: John Wiley and Sons (1979), Chapter 17. 


\section{Appendix I}

Marquardt's Minimization Procedure

The values of $G^{\prime}$ and $G^{\prime \prime}$ are obtained from eqs (6) and (7) using Marquardt's method [6, 7]. The method will be outlined below and the specific functions for our particular case will be given.

The Marquardt proccdurc is an efficient means of minimizing

$$
S(\mathbf{x})=\sum_{i=1}^{m}\left[\mathbf{f}_{i}(\mathbf{x})\right]^{2}=\mathbf{f}^{\top} \mathbf{f}
$$

where

$$
f_{i}(\mathbf{x})=g_{i}(\mathbf{x})-g_{i}^{\prime}
$$

and $g_{i}(\mathbf{x})$ are the $m$ (nonlinear) functions of the parameters $\mathbf{x}\left(x_{1}, x_{2}, \ldots x_{n}\right)$ and $g_{i}^{\prime}$ are the measured values. Equation (I-2) states that we are minimizing the sum of the squares of the deviation of the data from the functions used to fit these data. In our case, $m=2$ and $n=2$. The Marquardt procedure says that a good next guess for the value of the parameter $\mathbf{x}$, denoted $\mathbf{x}+\mathbf{q}$, can be determined from an iterative solution to

$$
\left(\mathbf{J}^{T} \mathbf{J}+\lambda \operatorname{Dia}\left[\mathbf{J}^{T} \mathbf{J}\right]\right) \mathbf{q}=-\mathbf{J}^{T} \mathbf{f}
$$

where $\mathbf{J}$ is the Jacobian matrix whose elements are defined as

$$
J_{i j}=\frac{\partial}{\partial x_{j}} f_{i}(\mathbf{x}) .
$$

The superscript $T$ denotes the transpose of the matrix and Dia [...] denotes that the matrix has all zero elements except along its diagonal. The left hand side of eq (I-4) must be positive definite and, therefore, $\lambda$ must always be chosen so that this side keeps its positive definiteness. The positivedefiniteness is determined in the solution to eq (I-3) by using the Choleski decomposition of the matrix on the left hand side and checking to see that each diagonal term of the decomposed matrix is greater than zero, which indicates positive-definiteness. If they are not all greater than zero, the value of $\lambda$ is increased by a factor of ten.

The iterative solution itself is straightforward. Starting with a value of $\lambda=0.1, \lambda$ is reduced by a factor of 2.5 before each step in the solution if the preceding solution for $\mathbf{q}$ has given

$$
S(\mathbf{x}+\mathbf{q})<S(\mathbf{x}) .
$$

If

$$
S(\mathbf{x}+\mathbf{q}) \geqslant S(\mathbf{x})
$$

then $\lambda$ is increased by a factor of 10 . The process is repeated until certain convergence criteria have been satisfied.

For our particular case we have from eq (6) that $x_{1}=G^{\prime}, x_{2}=G^{\prime \prime}, g_{1}=D^{\prime}$, and $g_{2}=\phi$, where, for convenience, we defined $D^{\prime}=\left(r_{b} / r_{t}\right) R$. Since $D^{\prime}$ and $\phi$ are functions of $x$ and $y$, which in turn are functions of $G^{\prime \prime}$ and $G^{\prime}$, it is easiest to use the chain rule for partial differentiation to obtain the four elements of the Jacobian matrix. Thus

$$
\begin{aligned}
& J_{11}=\frac{\partial D^{\prime}}{\partial G^{\prime}}=\frac{\partial D^{\prime}}{\partial x} \frac{\partial x}{\partial G^{\prime}}+\frac{\partial D^{\prime}}{\partial y} \frac{\partial y}{\partial G^{\prime}} \\
& J_{12}=\frac{\partial D^{\prime}}{\partial G^{\prime \prime}}=\frac{\partial D^{\prime}}{\partial x} \frac{\partial x}{\partial G^{\prime \prime}}+\frac{\partial D^{\prime}}{\partial y} \frac{\partial y}{\partial G^{\prime \prime}}
\end{aligned}
$$




$$
\begin{aligned}
& J_{21}=\frac{\partial \phi}{\partial G^{\prime}}=\frac{\partial \phi}{\partial x} \frac{\partial x}{\partial G^{\prime}}+\frac{\partial \phi}{\partial y} \frac{\partial y}{\partial G^{\prime}} \\
& J_{22}=\frac{\partial \phi}{\partial G^{\prime \prime}}=\frac{\partial \phi}{\partial x} \frac{\partial x}{\partial G^{\prime \prime}}+\frac{\partial \phi}{\partial y} \frac{\partial y}{\partial G^{\prime \prime}}
\end{aligned}
$$

where

$$
\begin{aligned}
\frac{\partial D^{\prime}}{\partial x} & =-\left[A^{2}+B^{2}\right]^{-3 / 2}\left[A \frac{\partial A}{\partial X}+B \frac{\partial B}{\partial x}\right] \\
\frac{\partial D^{\prime}}{\partial y} & =-\left[A^{2}+B^{2}\right]^{-3 / 2}\left[A \frac{\partial A}{\partial y}+B \frac{\partial B}{\partial y}\right] \\
\frac{\partial \phi}{\partial x} & =\left[A^{2}+B^{2}\right]^{-1}\left[A \frac{\partial B}{\partial x}-B \frac{\partial A}{\partial x}\right] \\
\frac{\partial \phi}{\partial y} & =\left[A^{2}+B^{2}\right]^{-1}\left[A \frac{\partial B}{\partial y}-B \frac{\partial A}{\partial y}\right]
\end{aligned}
$$

$A$ and $B$ are given by eq (7) and their derivatives are

$$
\begin{gathered}
\frac{\partial A}{\partial x}=\left[\left(C_{1}-1\right) \sin (x)+C_{1} x \cos (x)\right] \cosh (y)+C_{1} y \sin (x) \sinh (y) \\
\frac{\partial A}{\partial y}=\left[C_{1} x \sin (x)+\left(1-C_{1}\right) \cos (x)\right] \sinh y-C_{1} y \cos (x) \cosh (y) \\
\frac{\partial B}{\partial x}=-\frac{\partial A}{\partial y} \\
\frac{\partial B}{\partial y}=\left[C_{1} x \cos (x)+\left(C_{1}-1\right) \sin (x)\right] \cosh (y)+C_{1} y \sin (x) \sinh (y)
\end{gathered}
$$

The remaining partial derivatives are obtained from eq (4). Thus,

$$
\begin{gathered}
\frac{\partial x}{\partial G^{\prime}}=-\frac{1}{2 p^{2}}\left[x G^{\prime}-y G^{\prime \prime}\right] \\
\frac{\partial x}{\partial G^{\prime \prime}}=-\frac{1}{2 p^{2}}\left[x G^{\prime \prime}+y G^{\prime}\right] \\
\frac{\partial y}{\partial G^{\prime}}=\frac{\partial x}{\partial G^{\prime \prime}} \\
\frac{\partial y}{\partial G^{\prime \prime}}=-\frac{\partial x}{\partial G^{\prime}}
\end{gathered}
$$

Returning to eq (I-3) we can now write the solution for $q_{1}$ and $q_{2}$ in terms of the elements of the Choleski decomposed matrix $L_{i j}$ as

$$
\begin{gathered}
q_{2}=v_{1} / L_{22} \\
q_{1}=\left(v_{1}-L_{21} q_{2}\right) / L_{11}
\end{gathered}
$$

where 


$$
\begin{gathered}
v_{1}=b_{1} / L_{11} \\
v_{2}=\left(b_{2}-L_{21} v_{1}\right) / L_{22} \\
L_{11}=V A_{11} \\
L_{21}=A_{21} / A_{11} \\
L_{22}=A_{22}-A_{21}^{2} / A_{11}
\end{gathered}
$$

and

$$
\begin{gathered}
b_{1}=J_{11} f_{1}+J_{21} f_{2} \\
b_{2}=J_{12} f_{1}+J_{22} f_{2} \\
A_{11}=(1+\lambda)\left(J_{11}^{2}+J_{21}^{2}\right) \\
A_{12}=J_{11} J_{12}+J_{22} J_{21} \\
A_{22}=(1+\lambda)\left(J_{12}^{2}+J_{22}^{2}\right) \\
A_{21}=A_{21} .
\end{gathered}
$$

When $L_{22}>0$ the matrix is positive definite.

\section{Appendix II}

\section{Computation of the Mass Moment of Inertia $J$}

The total mass moment of inertia, $J$, is comprised of the following parts: (1) the base of the torsion spring; (2) the accelerometer mounting arm, including the accelerometer and the counter balance; (3) the specimen mounting plate; and (4) the Allen screw heads. Referring to figures A-1 to A-4, the following formulas and numerical values are obtained:

\section{Torsion Spring Base}

The mass moment of inertia, $J_{D}$, using figure A-1, is

$$
J_{2}=\frac{1}{2} W_{D}^{y} r_{D}^{2}
$$

where $W_{D}$ is the weight of the base and is given by

$$
W_{D}=\pi h_{D} r_{D}^{2} \rho
$$

where $\rho$ is the density of the base material. For steel, $W_{D}=0.09548 \mathrm{~kg}$ and $J_{D}=0.5825 \mathrm{~kg}-\mathrm{cm}^{2}$.

\section{Accelerometer Mounting Arm and Accelerometer}

The mass moment of inertia, $J_{5}$, using figure A. 2 is

$$
J_{2}=\frac{W_{c}}{2}\left(r_{0}^{2}+r_{i}^{2}\right)+2 W_{0}\left(\frac{L^{2}}{12}+d_{2}^{2}\right)
$$




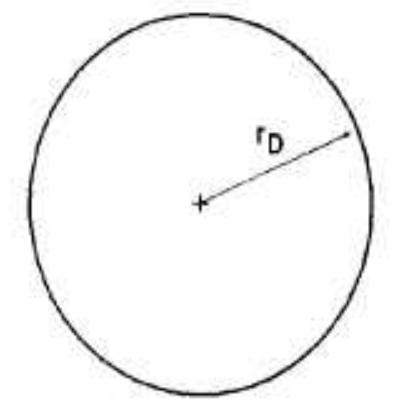

$$
\begin{aligned}
& r_{D}=34.93 \mathrm{~mm} \\
& h_{D}=3.18 \mathrm{~mm}
\end{aligned}
$$

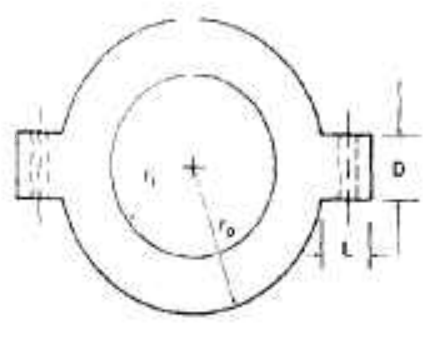

$r_{0}=34.93 \mathrm{~mm}$
$r_{i}=22.22 \mathrm{~mm}$
$r_{\mathrm{h}}=2.54 \mathrm{~mm}$
$d_{1}=47.63 \mathrm{~mm}$
$d_{2}=41.28 \mathrm{~mm}$
$D=19.05 \mathrm{~mm}$
$L=12.7 \mathrm{~mm}$
$h_{A}=6.35$ or $12.70 \mathrm{~mm}$

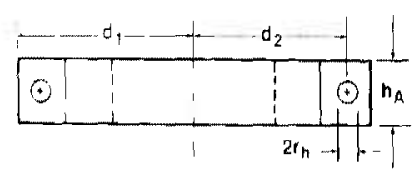

Figure A-2 - Dimensions of attached mass and top accelerometer's mounting position.

Figure A-1 - Dimensions of torsion spring base.

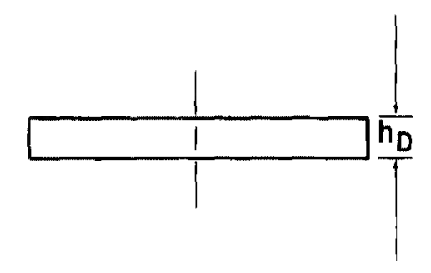

\section{- mounting position.}

where

$$
\begin{gathered}
W_{c}=\rho h_{A} \pi\left(r_{0}^{2}-r_{i}^{2}\right) \\
W_{0}=\rho h_{A}\left(L D-\pi r_{A}^{2}\right)+W_{\mathrm{ACC}}
\end{gathered}
$$

and $W_{\mathrm{ACC}}$ is the weight of the accelerometer $(2.5 \mathrm{gm})$. For aluminum, $W_{C}=\left(6.190 \times 10^{-3}\right) h_{A} \mathrm{~kg}$ and $W_{0}=\left(6.013 \times 10^{-4}\right) h_{A}+0.0025 \mathrm{~kg}$. Then for $h_{A}=6.35 \mathrm{~mm}, J_{S}=0.4680 \mathrm{~kg}-\mathrm{cm}^{2}$ and for $h_{A}=12.7 \mathrm{~mm}$, $J_{s}=0.9360 \mathrm{~kg}-\mathrm{cm}^{2}$.

\section{Specimen Mounting Plate}

The mass moment of inertia, $J_{M}$, using figure A-3, is

$$
J_{M}=\frac{1}{2}\left(W_{t} r_{t}^{2}+W_{b} r_{b}^{2}\right)
$$

where

$$
W_{i}=p h_{t} \pi r_{t}^{2}
$$

and

$$
W_{b}=\rho h_{b} \pi \mathrm{r}_{b}^{2}
$$

For aluminum, $W_{i}=0.03307 \mathrm{~kg}$ and $W_{b}=0.05355 \mathrm{~kg}$. Then $J_{M}=0.7308 \mathrm{~kg}-\mathrm{cm}^{2}$.

\section{Allen Screw Heads}

The mass moment of inertia, $J_{H}$, using figure A-4, is,

$$
J_{H}=4\left[W_{b}\left(\frac{1}{2} r_{c}^{2}+d_{3}^{2}\right)+W_{i}\left(\frac{1}{2}\left(r_{c}^{2}+r_{d}^{2}\right)+d_{3}^{2}\right)\right]
$$




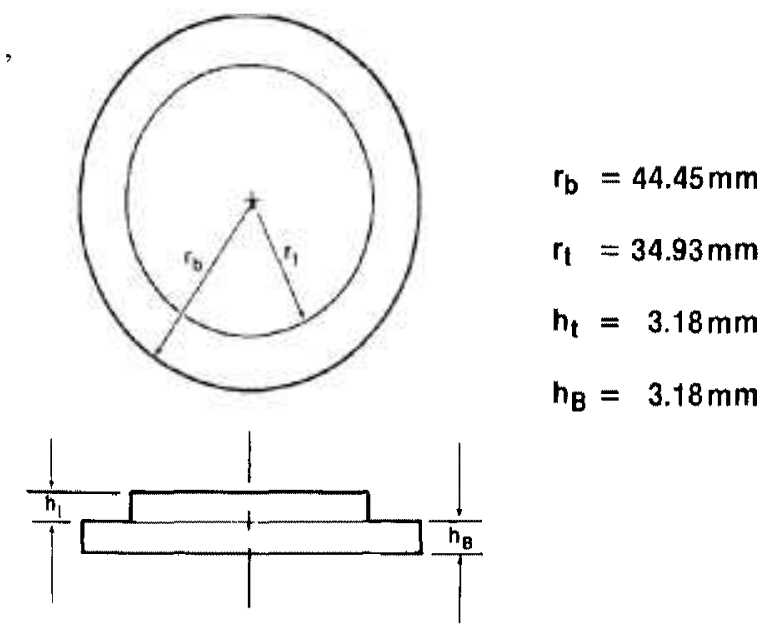

Figure A-3-Dimensions of specimen mounting plate.

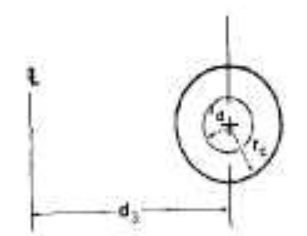

\section{LARGE}

$\mathrm{h}=3.18 \mathrm{~mm}$

$r_{d}=2.38 \mathrm{~mm}$

$r_{c}=4.37 \mathrm{~mm}$

$\mathrm{d}_{3}=28.58 \mathrm{~mm}$

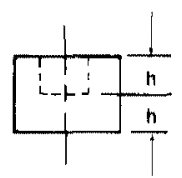

SMALL

$1.98 \mathrm{~mm}$

$2.38 \mathrm{~mm}$

$3.57 \mathrm{~mm}$

Figure A-4-Dimensions of Allen screw heads.

where

$$
W_{b}=\rho h \pi r_{c}^{2}
$$

and

$$
W_{t}=\rho h \pi\left(r_{r}^{2}-r_{d}^{2}\right) .
$$

For the large screws, $W_{b}=0.00149 \mathrm{~kg}$ and $W_{l}=0.00105 \mathrm{~kg}$. For the small screw, $W_{b}=0.000621 \mathrm{~kg}$ and $W_{t}=0.000345 \mathrm{~kg}$. Then, for the large screws $J_{H}=0.08408 \mathrm{~kg}-\mathrm{cm}^{2}$ and for the small screws $J_{H}=0.03185 \mathrm{~kg}-\mathrm{cm}^{2}$. The total is $J_{H}=0.1159 \mathrm{~kg}-\mathrm{cm}^{2}$.

\section{Total Mass Moment of Inertia}

The mass moment of inertia is equal to

$$
J=J_{D}+J_{S}+J_{M}+J_{H}
$$

For the two accelerometer mounting plates of thickness $h_{A}$ we have:

$$
\begin{aligned}
& h_{A}=6.35 \mathrm{~mm}: \\
& h_{A}=12.7 \mathrm{~mm}:
\end{aligned}
$$$$
J=1.8972 \mathrm{~kg}-\mathrm{cm}^{2}
$$$$
J=2.3652 \mathrm{~kg}-\mathrm{cm}^{2} \text {. }
$$ 